\title{
Efeitos do Uso Crônico de Acetaminofeno na Prenhez da Rata
}

\author{
Effects of the Chronic Use of Acetaminophen on Pregnant Rats
}

Jorge Andalaft Neto ${ }^{1}$, Manuel J. Simões ${ }^{2}$, Joaquim Evêncio Neto², Luiz Kulay Júnior ${ }^{2}$

\begin{abstract}
RESUMO
Objetivos: estudar a ação do acetaminofeno administrado durante toda a prenhez da rata albina.

Métodos: 40 ratas albinas prenhes foram distribuidas ao acaso em quatro grupos numericamente iguais: GI - recebeu diariamente $1 \mathrm{ml}$ de água destilada por gavagem (controle); GII, GIII e GIV foram tratadas, respectivamente, com 125, 500 e $1.500 \mathrm{mg} / \mathrm{kg}$ de peso corporal de acetaminofeno, dose única, diária, por gavagem, sempre diluído em $1 \mathrm{ml}$ de água destilada, desde o dia zero até o $20^{\circ}$ dia de prenhez. O ganho de peso das matrizes foi avaliado nos dias $0,7,15$ e 20 de prenhez, sendo que no $20^{\circ}$ dia todos os animais foram sacrificados. Resultados: nossos achados mostraram sempre menor peso entre as matrizes que receberam o medicamento, quando comparadas com o controle. A incidência de reabsorção dos embriões foi da ordem de 2,0, 3,5 e 7,0 vezes maior que no controle, considerando-se, respectivamente, os grupos GII, GIII e GIV. Os grupos GIII e GIV tiveram redução do peso dos conceptos. No GIV, houve redução de $50 \%$ no crescimento de fetos e placentas com relação ao controle; aqui, também foram encontradas 15,7\% de malformações externas.

Conclusões: deve ser evitado o uso contínuo do acetominofeno em altas doses, superiores a $70 \mathrm{mg} / \mathrm{kg}$ por dia, durante o periodo gestacional.
\end{abstract}

PALAVRAS-CHAVE: Drogas na gravidez. Placenta. Pré-natal.

\section{Introdução}

Aproximadamente $70 \%$ das gestantes, sem qualquer patologia, utilizam analgésicos para mitigar a dor conseqüente às modificações impostas

\footnotetext{
${ }^{1}$ Disciplina de Ginecologia e Obstetrícia da Faculdade de Medicina de Santo Amaro (UNISA), São Paulo.

${ }^{2}$ Departamento de Tocoginecologia da Universidade Federal de São Paulo/Escola Paulista de Medicina, São Paulo. Correspondência:

Luiz Kulay Júnior

Rua Napoleão de Barros, 715; $8^{\circ}$ andar.

Hospital São Paulo, Disciplina de Obstetrícia.

04024-002 - São Paulo-SP - Brasil.
}

pela gravidez ao organismo materno ${ }^{2,10}$. Entre os derivados do paraminofeno, o paracetamol ou acetaminofeno é, a par dos salicilatos, o analgésico mais utilizado em todo o mundo, inclusive durante a gestação, em razão dos poucos efeitos colaterais, em doses terapêuticas ${ }^{1,7}$.

No campo da experimentação, o uso de doses elevadas em ratas, ainda que por curtos periodo de tempo nos vários estágios da prenhez, demonstrou efeitos adversos do acetaminofeno sobre o binômio materno-fetal. Foram relatados diminuição tanto do ganho de peso $^{9}$, quanto a ocorrência de necrose hepática e aumento do índice de mortalidade ${ }^{3}$ das matrizes. No que diz respeito 
aos conceptos, foram observados aumento de reabsorções e diminuição de peso ${ }^{5}$, constricção do ducto arterial ${ }^{6}$ e malformações ${ }^{12}$.

Tais fatos motivaram-nos a ministrar o acetaminofen durante toda a prenhez da rata para avaliar os seus efeitos sobre o binômio materno-fetal.

\section{Material e Métodos}

\section{Animais e tratamento}

Utilizamos ratas (Wistar EPM-1), adultas, virgens, pesando de 150 a $200 \mathrm{~g}$, com regime de alimentação (ração Purina-Labina) e de água à vontade. Foram inicialmente acasaladas na proporção de duas fêmeas para cada macho por um período de duas horas. O teste de prenhez foi determinado pelo encontro de espermatozóides na luz vaginal. Quarenta ratas foram então divididas ao acaso em quatro grupos numericamente iguais, a saber: I, animais que receberam água (controle); II, animais tratados com $125 \mathrm{mg} / \mathrm{kg}$ de acetaminofeno (paracetamol - substância gentilmente cedida pelo Laboratório Johnson e Johnson, com teor de pureza 99\%); III, animais tratados com $500 \mathrm{mg} / \mathrm{kg}$ de acetaminofeno e IV, animais tratados com 1.500 $\mathrm{mg} / \mathrm{kg}$ de acetaminofeno. O tratamento teve início logo após a positividade do teste de prenhez (dia 0) e se estendeu até o $20^{\circ}$ dia, sendo que cada um dos animais de qualquer dos grupos estudados recebeu diariamente sempre o volume total de $1 \mathrm{ml}$ de solução, por gavagem, independente do grupo apreciado.

Todas as ratas foram pesadas nos dias 0 , 7,15 e 20 de prenhez. O ganho de peso percentual foi calculado de acordo com a seguinte fórmula:

$$
\begin{aligned}
\Delta \%= & (\text { peso no } \underline{X} \text { dia }- \text { peso inicial }) \times 100 / \text { peso inicial } \\
& \text { onde } \underline{X}=7^{\circ} \text { ou } 15^{\circ} \text { ou } 20^{\circ} \text { dia de prenhez. }
\end{aligned}
$$

Ao termo $\left(20^{\circ} \mathrm{dia}\right)$, todos os animais foram sacrificados sob anestesia profunda com éter etílico; após laparotomia seguida de histerotomia, foram anotados os seguintes parâmetros: número de sítios de implantação e de reabsorção, número de placentas e de fetos, determinados os pesos das placentas e dos fetos e malformações maiores (cardiovasculares, hipospádia, fenda palatina/lábio leporino, espina bífida, encurtamento de membros e polidactilia).

\section{Análise estatística}

Análises paramétricas e não-paramétricas foram realizadas; o nivel de rejeição para hipótese de nulidade foi fixada em 5\%. Múltiplas comparações foram feitas pelo teste de KruskalWallis e análise de variância.

\section{Resultados}

Os ganhos de peso das ratas foram significantemente menores nos animais que receberam acetaminofeno em qualquer das doses utilizadas ou seja 125, 500 e $1.500 \mathrm{mg} / \mathrm{kg}$ de peso corporal por dia, como pode ser visto na Figura 1.

Observamos diminuição dos pesos individuais dos fetos e das ninhadas quando expostos a 500 e $1.500 \mathrm{mg} / \mathrm{kg}$ de peso por dia. O número de fetos vivos, placentas e o peso individual médio das placentas encontravam-se diminuídos somente no grupo IV, que recebeu $1.500 \mathrm{mg}$ de acetaminofen por $\mathrm{kg}$ de peso durante todo o curso da prenhez. Foram encontradas malformações, redução do comprimento de membros e polidactilia em todos os grupos tratados, principalmente quando os conceptos foram expostos a $1.500 \mathrm{mg} / \mathrm{kg}$ por dia de acetaminofeno (Tabela 1 ).

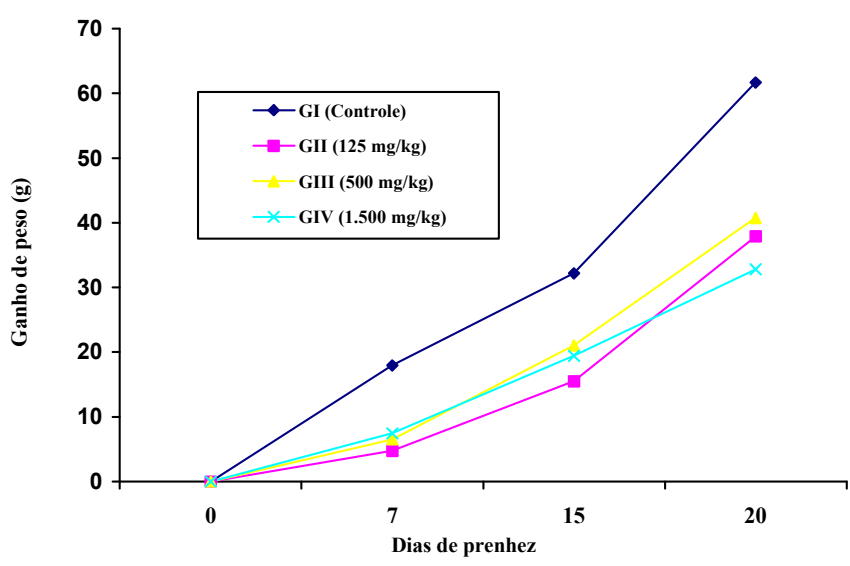

Figura 1 - Efeitos do acetaminofeno sobre o ganho de peso, $(\Delta \%)$ nos vários períodos estudados, na rata prenhe. ( $\mathrm{Gl} \neq$ dos outros, aos 7,15 e 20 dias de prenhez; $\mathrm{p}<0.05)$. 
Tabela 1 - Efeitos do acetaminofeno sobre os vários parâmetros estudados na rata (média $\pm \mathrm{DP}$ )

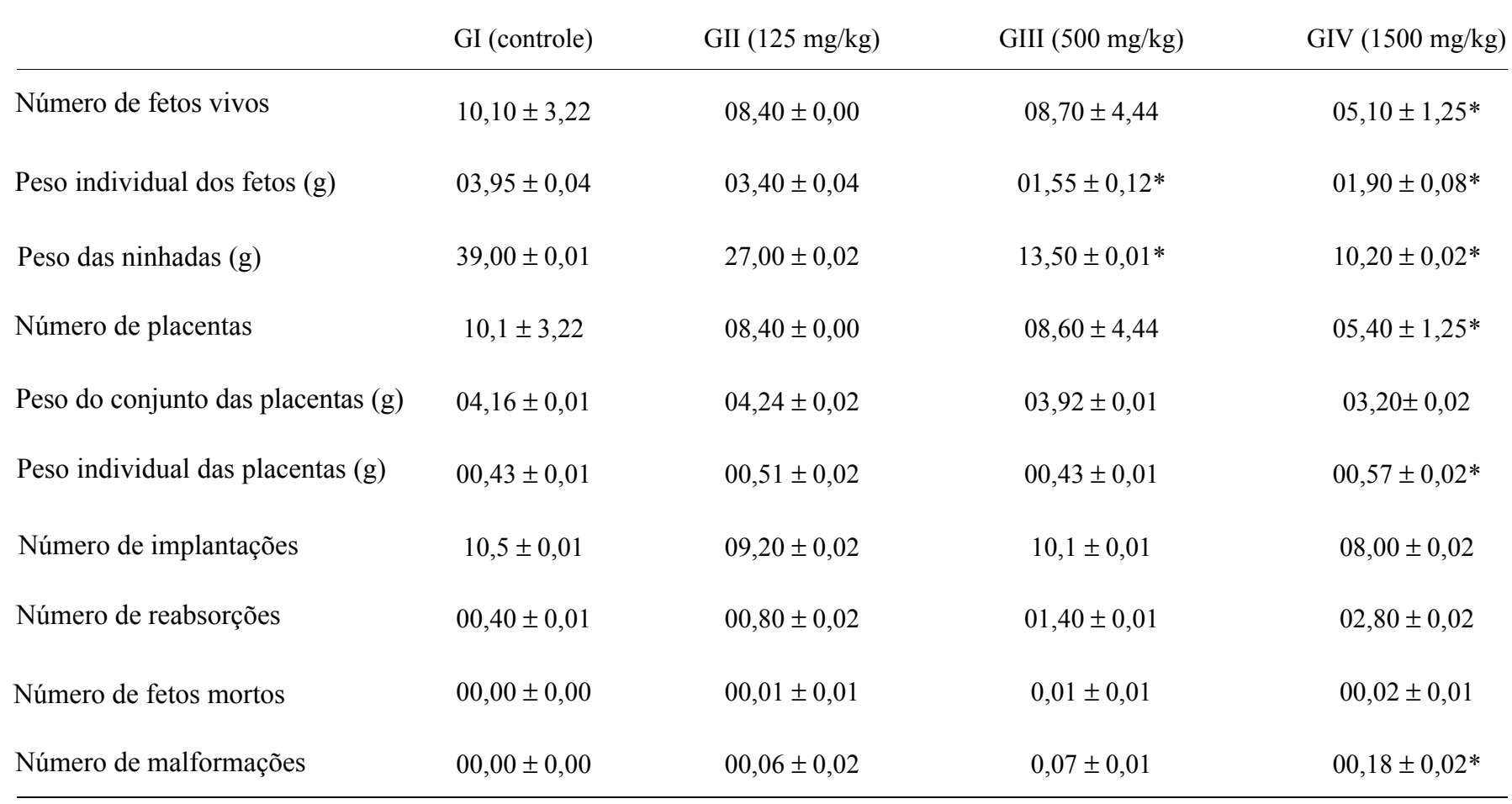

* Significantemente diferente dos outros grupos $(\mathrm{p}<0,05)$.

\section{Discussão}

Nossos resultados mostraram que houve aumento do ganho de peso corporal de todas as matrizes aqui estudadas, no decorrer da prenhez. Esse ganho, no entanto, foi menor nos grupos que receberam acetaminofeno, independentemente da dose administrada. Esses achados confirmam, assim, os trabalhos de Renault et al. ${ }^{9}$ e Wright ${ }^{14}$ que referem ainda ser a droga lesiva para o organismo materno. Observamos ainda que os conceptos expostos a doses superiores a DL 50 - $338 \mathrm{mg} / \mathrm{kg}$, via oral - ou seja, igual ou maior que $500 \mathrm{mg} / \mathrm{kg}$ de peso corporal por dia, tiveram seu peso diminuído, o que está de acordo com o relato de Lubawy e Garrett ${ }^{5}$. Ministrando dose bem mais elevada, $1.500 \mathrm{mg} / \mathrm{kg}$ de peso corporal por dia, houve redução do número de fetos vivos e, conseqüentemente, do número de placentas além de elevada incidência de malformações maiores, achados esses que se assemelham aos resultados de Ying e Lou ${ }^{15}$.

É interessante lembrar que o acetaminofeno possui efeito embriotóxico. Quando acrescentado na dose de $300 \mathrm{mg} / \mathrm{ml}$ a um meio de cultura de tecido causa aumento da freqüência de micronúcleos, redução do número de células e alterações morfológicas dos blastocistos ${ }^{12,15}$. Esses dados, nos parece, estão diretamente relacionados ao aumento significante de malformações maiores, mas não atendem outros achados.

A ação do acetaminofeno, no entanto, é mais ampla. Adicionado na dose de $10 \mathrm{mg} / \mathrm{ml}$ em meio de cultura de células endoteliais diminui a produção de prostanóides, principalmente PGE2, que é o responsável pela manutenção do tônus muscular ${ }^{8}$. Sem a ação protetora da PGE2, o rim da rata apresenta diminuição das taxas de filtração glomerular e de reabsorção de glicose ${ }^{11}$. No entender de Whelton ${ }^{13}$, a maioria das síndromes renais provocadas por antiinflamatórios não-hormonais é devida à inibição das prostaglandinas. No que se refere aos figados de rato, 36 horas após ingestão de $600 \mathrm{mg} / \mathrm{kg}$ de peso corporal, podem ser observados espaços centrolobulares com áreas irregulares e lesões microvasculares nessa região como também ausência de sinusóides periportais ${ }^{4}$.

Tais fatos, ao que tudo indica, são suficientes para justificar a redução tanto do número de nativivos quanto de seus respectivos pesos. Além do comprometimento específico das funções renal 
e hepáticas, que prejudicam a detoxificação orgânica, a vasoconstricção universal, em grau maior ou menor, com toda certeza, também interfere na homeostase fetal. Deve ser evitado, portanto, o uso contínuo do acetaminofeno em altas doses, a partir de $70 \mathrm{mg} / \mathrm{kg}$ de peso por dia durante o periodo gestacional.

\section{SUMMARY}

Purpose: to evaluate the effects of acetaminophen on the pregnancy of female albino rats.

Methods: forty pregnant rats were separated into four groups. All the animals received daily by gavage $1 \mathrm{ml}$ of acetaminophen solution from the first day (day zero) until the 20th day of pregnancy: group I - only distilled water (control); groups II, III and IV, respectively, 125, 500 and $1,500 \mathrm{mg} / \mathrm{kg}$ body weight of acetaminophen dissolved in distilled water. The animals were weighed on days 0, 7, 15 and 20 of pregnancy.

Results: our results showed that the rats that received the medication presented a reduction in weight when compared to the control group. The incidence of reabsorption of the embryos was 2.0, 3.5 and 7.0 times higher than in the control, in groups II, III and IV, respectively. Groups GII and GIV showed a clear reduction in the weight of the concepts. In GIV there was a $50 \%$ reduction in weight increase of fetuses and placentas when compared to the control, and $15.7 \%$ of external malformations were also found.

Conclusions: the continuous use of acetaminophen should be avoided at doses higher than $70 \mathrm{mg} / \mathrm{kg}$ per during pregnancy.

KEY WORDS: Acetaminophen. Placenta. Prenatal care. Pregnancy, drugs in.

\section{Referências}

1. Dahlin DC, Nelson SD. Synthesis, decomposition, kinetic, and preliminary toxicological studies of pure $\mathrm{N}$-acetyl-p-benzoquinone imine, a proposed toxic metabolite of acetaminophen. J Med Chem 1982; 25: 885-6.
2. Deláscio D, Guariento A, editores. Obstetrícia normal Briquet. 3 ed. São Paulo: Sarvier; 1981. p. 139-74.

3. Larrey D, Letteron P, Foliot A, Descatoire V, Degott C, Geneve J, et al. Effects of pregnancy on the toxicity and metabolism of acetaminophen in mice. J Pharmacol Exp Ther 1986; 237: 283-91.

4. Lim SP, Andrews FJ, O’Brien PE. Acetaminophen induced microvascular injury in the rat liver: protection with misoprostol. Hepatology 1995; 22: 1776-81.

5. Lubawy WC, Garrett BRJ. Effects of aspirin and acetaminophen on fetal and placental growth in rats. J Pharm Sci 1977; 66: 111-3.

6. Momma K, Takao A. Transplacental cardiovascular effects of four popular analgesics in rats. Am J Obstet Gynecol 1990; 162: 1304-10.

7. Niederhoff H, Zahradnik H. Analgesics during pregnancy. Am J Med 1983; 14: 117-20.

8. O'Brien WF, Krammer J, O'Eary TD, Mastrogiannis DS. The effect of acetaminophen on prostacyclin production in pregnant women. Am J Obstet Gynecol 1993; 168: 1164-9.

9. Renault H, Rohrbach P, Dugniolle J. Propriétés pharmacodynamiques du N-acéthylparaminophénol, métabolite de la phénacetine et de 1'acétanilide. Thérapeutique 1956; 11: 300-7.

10.Rezende J, editor. Obstetrícia. $3^{\text {a }}$ ed. Rio de Janeiro: Guanabara Koogan; 1974. p. 130-46.

11.Trumper L, Monasterolo LA, Ochoa E, Elias MM. Tubular effects of acetaminophen in the isolated perfused rat kidney. Arch Toxicol 1995; 69: 248-52.

12.Weeks BS, Gamache P, Klein NW, Hinston JA, Bruno $\mathrm{M}$, Khairallah E. Acetaminophen toxicity to cultured rat embryos. Teratog Carcinog Mutagen 1990; 10: 361-71.

13. Whelton A. Renal effects of over-the-counter analgesics. J Clin Pharmacol 1995; 35: 454-63.

14.Wright HN. Chronic toxicity studies of analgesic and antipyretic drugs and congeners. Toxicol Appl Pharmacol 1967; 11: 280-92.

15.Ying Y, Lou YJ. Effects of preimplantation treatment with aspirin and acetaminophen on blastocyst and fetus in rats. Chung Kuo Yao Li Hsueh Pao 1993; 14: 369-72. 